\title{
Comparative Evaluation of Calcium-Sensitizing Agents, Pimobendan and SCH00013, on the Myocardial Function of Canine Pacing-Induced Model of Heart Failure
}

\author{
Lina Hamabe ${ }^{1, *}$, Keisuke Kawamura ${ }^{1}$, Soo-min Kim ${ }^{1}$, Rieko Yoshiyuki ${ }^{1}$, Toshiharu Fukayama ${ }^{1}$, \\ Miki Shimizu $^{2}$, Ryuji Fukushima ${ }^{1}$, and Ryo Tanaka ${ }^{1}$ \\ ${ }^{1}$ Department of Veterinary Surgery, ${ }^{2}$ Veterinary Diagnostic Imaging, Faculty of Veterinary Medicine, \\ Tokyo University of Agriculture and Technology, Saiwaicho 3-5-8, Fuchu, Tokyo 183-8509, Japan
}

Received October 8, 2013; Accepted January 17, 2014

\begin{abstract}
Pimobendan and SCH00013 are calcium sensitizers that possess dual action of calcium sensitization and phosphodiesterase-III inhibition. This study was conducted to comparatively evaluate the effect of these medications on the myocardial function of the canine pacing-induced heart failure model using echocardiography. Heart failure was induced in 20 dogs, to which pimobendan and two different doses of SCH00013 were administered orally to 15 dogs for 3 weeks, and the remaining 5 dogs served as the control. Cardiac evaluations were performed at baseline, week 1, week 2, and week 3 . Significant thinning and dilation of the left ventricles, with systolic dysfunction, indicated by reduction of fractional shortening (FS) and strain values, were observed with a low dose of SCH00013. Whereas, although systolic dysfunction was observed with reduction of FS and radial strain, significant dilation and thinning of the left ventricles and reduction of circumferential strain were not observed with pimobendan. Pimobendan had a potent positive inotropic effect, with little effect on synchronicity, while low-dose SCH00013 had a weaker positive inotropic effect but was able to sustain synchronicity. Although, it failed to show significant statistical differences, the results of this study allow speculations that administration of pimobendan and SCH00013 may have differing effect on the myocardial function in the canine pacing-induced heart failure model.
\end{abstract}

Keywords: animal model, calcium sensitizer, echocardiography, heart failure, inotropic agent

\section{Introduction}

Inotropic agents have been indicated for the treatment of congestive heart failure (CHF) with reduced cardiac output and peripheral hypo-perfusion $(1,2)$. Classic inotropic agents, including cardiac glycosides, catecholamines, and phosphodiesterase (PDE) III inhibitors, improve impaired cardiac pump function by elevating intracellular calcium concentration in myocardial cells to directly improve the myocardial contractility $(2-4)$. While they effectively enhance the cardiac pump function, these agents have also been associated with major side effects such as cardiac arrhythmia, induction of

*Corresponding author. linahamabe@vet.ne.jp

Published online in J-STAGE on March 4, 2014

doi: 10.1254/jphs.13196FP myocardial cell injury, and unfavorable increase of myocardial energy consumption $(2-4)$.

Calcium sensitizer is a relatively new class of inotropic agents, which enhance myocardial contractility by increasing sensitivity of contractile protein to calcium $(2-4)$. Unlike the classic inotropic agents, these agents augment the affinity of calcium to contractile proteins, resulting in more force to be generated for a given intracellular calcium concentration $(2-4)$. Advantages include lack of intracellular calcium overload and preserved energy consumption, and additionally the ability of the myofilaments to respond to calcium sensitization is preserved in heart failure (HF) $(2-4)$. Most of these agents are not pure calcium-sensitizers and possess additional mechanisms of action (4).

Pimobendan, a benzimidazole-pyridazinone derivative, elicits its positive inotropic effect (PIE) by 1) calcium 
sensitizing action by increasing the affinity of the regulatory site on troponin $\mathrm{C}$ for calcium and 2) PDE III inhibitory action $(5-8)$. The PIE of pimobendan is mostly due to a cAMP-dependent mechanism, and the calcium-sensitizing action plays a minor role (6). SCH00013, a pyridazinone derivative, also possesses both actions of calcium sensitization by increasing myofilament calcium sensitivity and PDE III inhibition $(9-11)$. However in contrary to pimobendan, calciumsensitizing action is the main contributor of $\operatorname{PIE}(10,12)$. The effects of these two calcium sensitizers have not been comparatively evaluated in a clinical model of HF. Although these two agents possess both actions of calcium sensitization and PDE II inhibition, they differ in action mechanism, including the proportion of the contributing actions of PIE. Therefore, we hypothesized that pimobendan and $\mathrm{SCH} 0013$ would have a different effect on the myocardial function in HF. This study was undertaken to assess the effect observed with oral administration of pimobendan and SCH00013 on the myocardial function of the canine pacing-induced HF model using echocardiography.

\section{Materials and Methods}

\section{Study design}

Twenty female Beagles were used to create pacinginduced HF model, and of these dogs, 15 received medication and the remaining 5 served as the control. Treatment included pimobendan (Vetmedin; Nippon Boehringer Ingelheim Co., Ltd., Tokyo), and two different doses of SCH00013 (Zenyaku Kogyo Co., Ltd., Tokyo), with 5 dogs in each treatment group. Implantation of an external pacemaker lead (TF204-062; KMY Medical, Inc., Tokyo) to the right ventricular apex was performed via thoracotomy as described in a previous report, and pacing-induced HF was created by sustained high-electrical pacing of $250 \mathrm{bpm}$ for 3 consecutive weeks (13). Administration of medications was initiated from the first day of pacing in the following treatment groups: pimobendan $(0.25 \mathrm{mg} / \mathrm{kg}$ PO q12hrs), Low-SCH (SCH00013, $1 \mathrm{mg} / \mathrm{kg}$ PO q12hrs), and High-SCH (SCH00013, $3 \mathrm{mg} / \mathrm{kg}$ PO q12hrs). The recommended total daily dosage of $0.5 \mathrm{mg} / \mathrm{kg}$ of pimobendan used for the treatment of canine heart failure, and total daily dosage of $2 \mathrm{mg} / \mathrm{kg}$ and $6 \mathrm{mg} / \mathrm{kg}$ of SCH00013 for cardioprotective effect and PIE, respectively, were used, which were established by the manufacturer. Cardiac evaluation including indirect oscillometric blood pressure (BP) exam (BP-100D; FUKUDA M.E., Tokyo), standard 6-lead ECG ( $\alpha 8000$, FUKUDA M.E.), conventional echocardiography, and two-dimensional tissue tracking analysis were performed at the baseline and weeks 1,2, and 3 of pacing, for a total of 4 occasions. The entirety of the evaluation was performed during a temporary cessation of pacing, at least an hour after the pacemaker was turned off to minimize the influence of pacing. All of the pacemakers were checked for accurate pacing at the end of each evaluation. The study was carried out in accordance with the standards established by the Tokyo University of Agriculture and Technology and as described in its Guide for the Care and Use of Laboratory Animals. All experimental procedures were approved by the Animal Experimental Committee of Tokyo University of Agriculture and Technology (acceptance No. 21-30).

\section{Echocardiography}

An ultrasonography unit (ALOKA prosound $\alpha 10$; Hitachi Aloka Medical, Ltd., Tokyo) equipped with a $5-\mathrm{MHz}$ phased array transducer probe (UST52108, Hitachi Aloka Medical) was used. All echocardiographic examinations were performed with concurrent ECG monitoring and by the same trained examiner for all dogs. All examinations were performed on conscious dogs, using only gentle restraint. A mean of 5 measurements of each parameter was obtained from 5 consecutive cardiac cycles.

\section{Conventional echocardiography}

The examinations were performed in accordance with the methods described by Boon (14). Left ventricular (LV) measurements were taken from the right parasternal short axis view at the level of the papillary muscle using M-mode. Measurements included LV end-diastolic (LVIDd) and end-systolic (LVIDs) diameters, interventricular septal thickness in diastole (IVSd) and in systole (IVSs), LV free wall thickness in diastole (LVPWd) and in systole (LVPWs), and fractional shortening (FS). Additionally, diastolic parameters including early (E) and late (A) ventricular filling velocity, E/A ratio, and deceleration time were obtained from the trans-mitral flow profile at left parasternal apical four chamber view. Early diastolic myocardial velocities ( $\left.E^{\prime}\right)$ were measured at IVS and LVPW of mitral annulus in the left apical views using pulsed tissue Doppler imaging, and $\mathrm{E} / \mathrm{E}^{\prime}$ was also calculated.

\section{Two-dimensional tissue tracking}

Right parasternal short axis view at the level of the papillary muscle with the frame rate of $70-110$ frames/s were acquired and saved onto a hard disk, which was then analyzed off-line (DAS-RS1 software 1.1v, Hitachi Aloka Medical). Strain analysis involved multiple steps. Initially, the endocardium and the epicardium were manually traced at end-systole by placing a number of 
points at the borders of the myocardium. The software then automatically tracked these points on a frameby-frame basis and divided the LV into 6 segments (anterior, lateral, posterior, inferior, septal, and anterior septal). Regional peak systolic strains of the 6 segments and global peak systolic strains, which is the mean of the 6 segments, for both radial and circumferential directions were calculated. LV synchrony was also assessed by calculating the synchrony time index (STI), which is the difference in timing of peak strains from the earliest to the latest segments in the radial direction.

\section{Statistical analysis}

Variables are expressed as the mean \pm standard deviation. Normality of the variables was tested using the Kolmogorov-Smirnov test and the visual inspection of histograms. Clinical and echocardiographic variables were compared across 4 time points using one-way repeated-measures ANOVA for parametric variables, and the Friedman test for non-parametric variables. Once significant difference was detected, Dunn's Multiple Comparison test was used to further compare each time point with the baseline. Additionally, two-way ANOVA in conjunction with Bonferroni's multiple comparison tests was used to detect significant difference among groups using the percent change from the baseline. Significant difference was defined as $P<0.05$. Statistical analyses were performed using statistical software (Prism 5.0v, from GraphPad Software, Inc., La Jolla, CA, USA and JMP 10.0.2, from SAS Institute Inc., Cary, NC, USA).

\section{Results}

The pacemaker implantation was successful and the recovery was uneventful in all 20 dogs. They were able to maintain adequate physical condition and survived until the last day of pacing.

The resting heart rate with cessation of pacing at each examination revealed no significant changes from the baseline in all 4 groups. BP measurements showed declining trends in all 4 groups. However, significant reductions were only seen with the control and High$\mathrm{SCH}$ group. In the control group, significant reductions of mean arterial pressure (MAP) and diastolic pressure were observed from week 2 and week 3, respectively $(\mathrm{MAP}=0.001$, diastolic $=0.007)$; in the High-SCH group, significant reductions of systolic pressure and MAP were observed from week 1 and week 2 of pacing, respectively (systolic $P=0.002$, MAP $=0.02$ ) (Fig. 1). Additionally, no abnormalities on the ECG were observed at each examination.
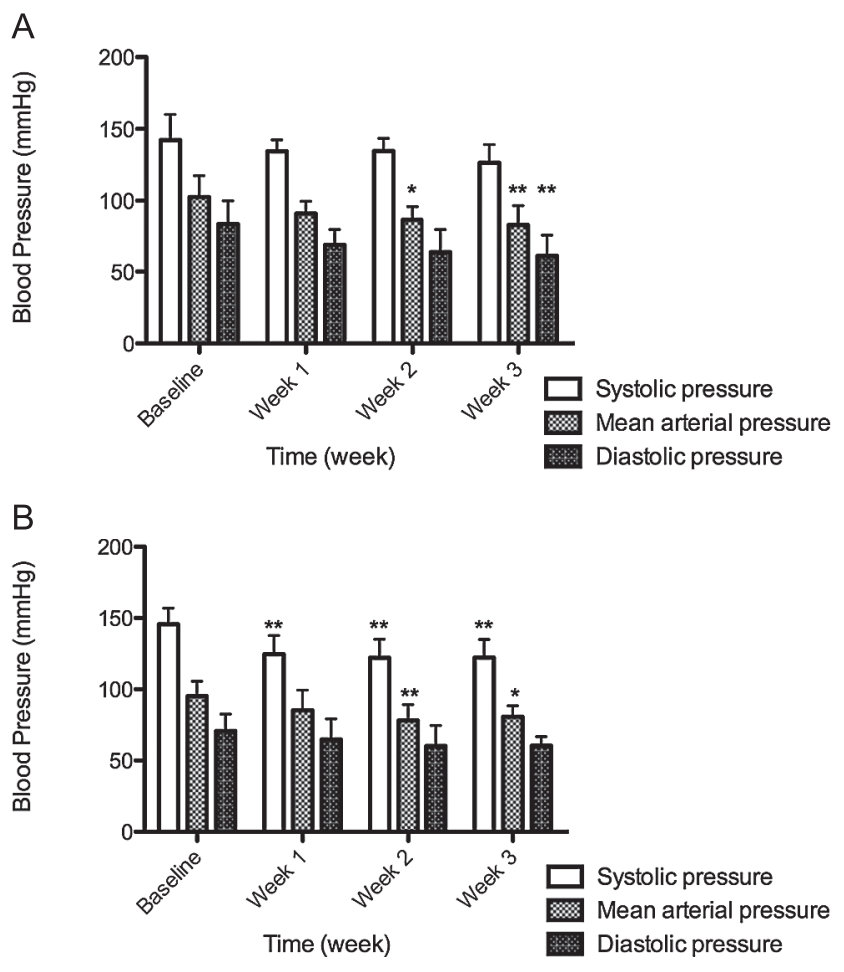

Fig. 1. Temporal changes of blood pressure measurements observed in the canine pacing-induced heart failure model (A) without treatment (control) and (B) with concurrent administration of high dose of $\mathrm{SCH} 00013$ (High-SCH) (1 and $3 \mathrm{mg} / \mathrm{kg}$ PO q12hrs).

\section{Conventional echocardiography}

Figure 2 shows examples of FS and strain analysis at baseline and week 3 of pacing. Sustained high-electrical pacing in the control had resulted in immediate alteration of LV morphology, including significant thinning of LV walls, with the exception of LVPWd, and significant dilation of LV from week 1 of pacing (Table 1). Additionally, significant reduction of FS was also observed from week 1. Furthermore, diastolic variables revealed significant reduction of $\mathrm{A}(P=0.02)$ at week 3 and deceleration time $(P=0.02)$ from week 2 of pacing, and $\mathrm{E}$, and no significant changes were observed with $\mathrm{E}, \mathrm{E} / \mathrm{A}$, and $\mathrm{E} / \mathrm{E}^{\prime}$ at IVS and LVPW.

Similar morphological changes of LV, including both significant dilation and thinning of $\mathrm{LV}$, and reduction of FS were observed in both Low- and High-SCH groups from week 1 of pacing, with the exception of IVSd in the High-SCH group. In regards to diastolic function, significant reduction of $\mathrm{A}(P=0.01)$ and deceleration time $(P=0.04)$ were briefly observed at week 2 in the Low-SCH group, but no other changes, including $\mathrm{E}$, $\mathrm{E} / \mathrm{A}, \mathrm{E} / \mathrm{E}^{\prime}$ at IVS, and LVPW, were observed in either treatment groups with $\mathrm{SCH} 00013$.

With the group treated by pimobendan, significant dilation and thinning of the LV was not evident, but 
A

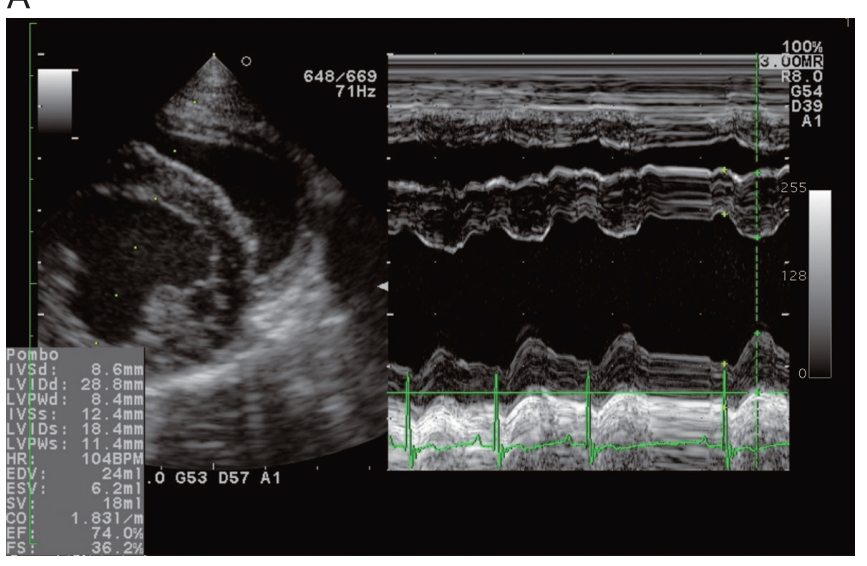

B

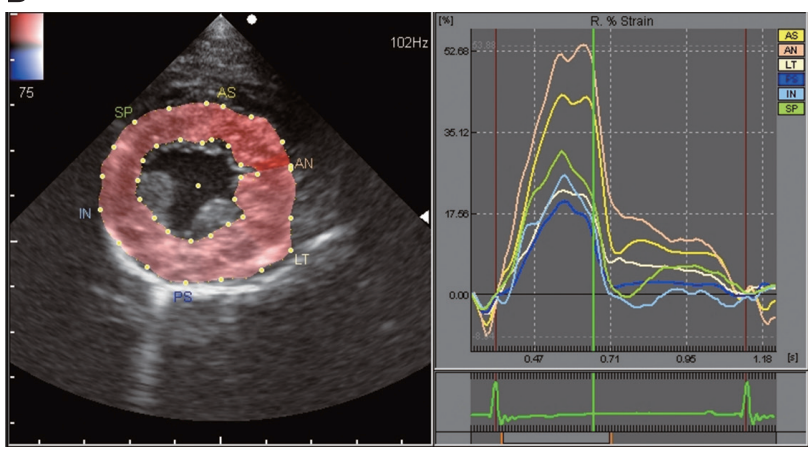

C

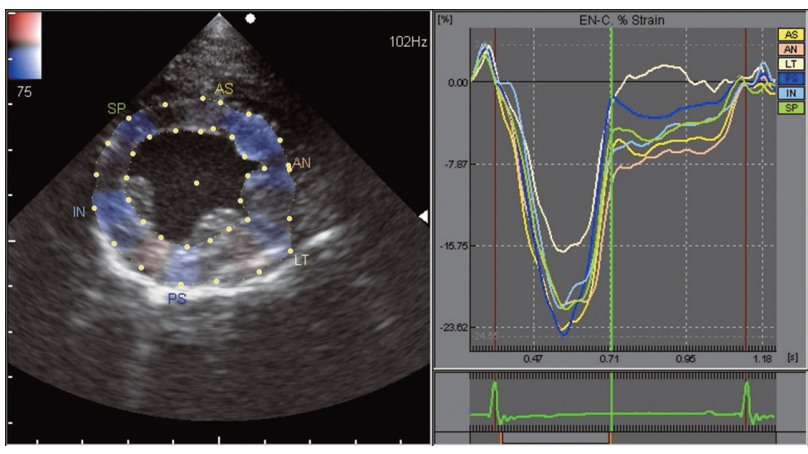

D

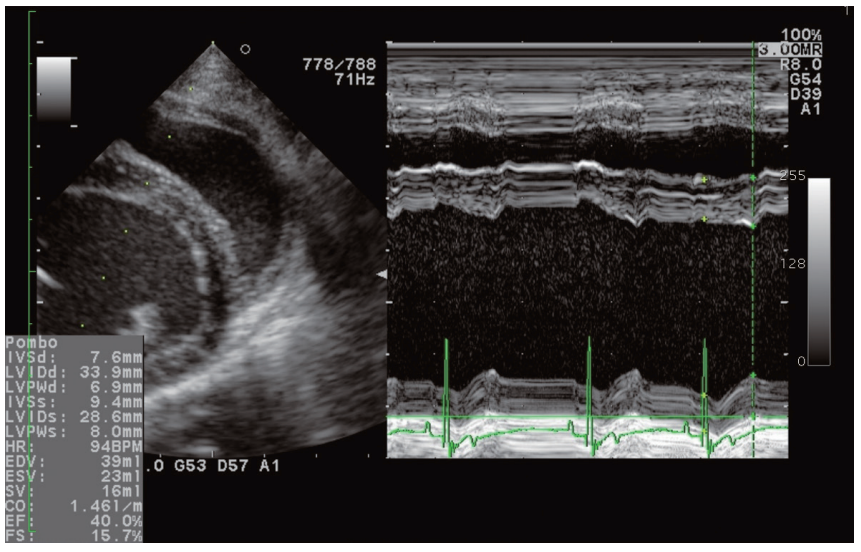

E

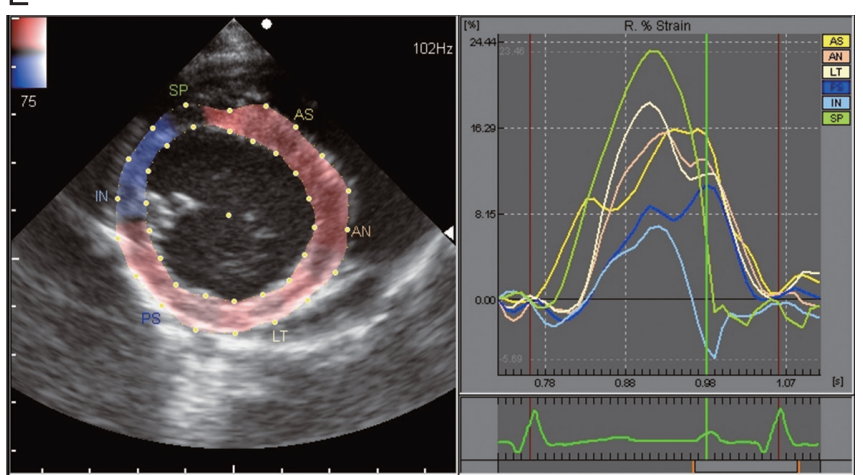

$\mathrm{F}$

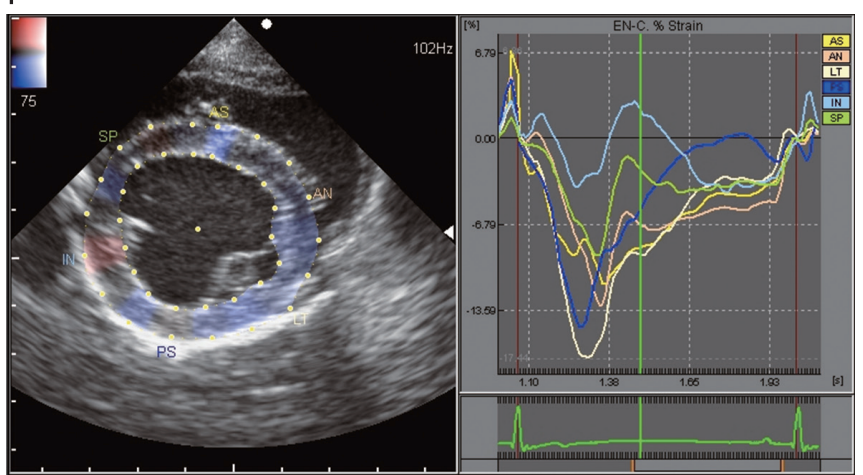

Fig. 2. Examples of fractional shortening (FS) and radial and circumferential strain profiles obtained from the right parasternal short axis view at the level of the papillary muscle using the M-mode and two-dimensional tissue tracking, respectively. Radial strain represents thickening and thinning motion of the myocardium in the radial direction, and circumferential strain represents myocardial motion along the circular perimeter. In the systole, the myocardial deformation increases in the radial direction, thus the radial strain becomes positive, and the myocardial deformation decreases in the circumferential direction, causing the circumferential direction to becomes negative. FS (A) and radial (B) and circumferential (C) strains at baseline, and FS (D) and radial (E) and circumferential (F) strains at week 3 of sustained high electrical pacing.

showed significant reduction of FS from week 1 of pacing. Additionally, no significant change to the diastolic variables was observed.

Significant difference in the degree of LV dilation was observed between the groups. Significant differences in LVIDd $(P=0.001)$ were observed between the pimobendan group and control and High-SCH group from week 2, and between the pimobendan group and Low-SCH group at week 2 only. Additionally, significant difference between the control and Low-SCH group was seen at week 3 . For LVIDs $(P=0.03)$, significant difference was observed between the control and pimobendan group from week 2 . 
Table 1. Temporal comparison of the M-mode parameters in the control and those with the administration of pimobendan $(0.25 \mathrm{mg} / \mathrm{kg}$ PO q12hrs) and two doses of SCH00013 (1 and $3 \mathrm{mg} / \mathrm{kg}$ PO q12hrs) on of canine myocardial dysfuncion model

\begin{tabular}{|c|c|c|c|c|c|}
\hline & & Baseline & Week 1 & Week 2 & Week 3 \\
\hline \multirow[t]{4}{*}{ LVIDd (mm) } & Control & $29.40 \pm 3.08$ & $33.09 \pm 3.45^{* *}$ & $36.87 \pm 2.84 * * *$ & $39.18 \pm 4.84^{* * *}$ \\
\hline & Pimobendan & $33.82 \pm 14.81$ & $33.68 \pm 14.34$ & $31.51 \pm 13.93$ & $35.03 \pm 14.52$ \\
\hline & Low-SCH & $31.70 \pm 13.18$ & $35.90 \pm 14.89 * *$ & $36.38 \pm 15.02 * * *$ & $37.34 \pm 15.44 * * *$ \\
\hline & High-SCH & $31.02 \pm 12.80$ & $34.00 \pm 14.18^{*}$ & $35.86 \pm 14.90^{* *}$ & $37.62 \pm 15.61 * * *$ \\
\hline \multirow[t]{4}{*}{ LVIDs (mm) } & Control & $19.22 \pm 3.03$ & $26.49 \pm 3.03 * * *$ & $30.00 \pm 2.11 * * *$ & $32.16 \pm 3.87 * * *$ \\
\hline & Pimobendan & $21.26 \pm 9.72$ & $26.01 \pm 11.33^{*}$ & $24.04 \pm 11.48$ & $25.74 \pm 10.80$ \\
\hline & Low-SCH & $20.14 \pm 8.61$ & $26.14 \pm 10.93 * *$ & $27.80 \pm 11.68 * * *$ & $28.88 \pm 12.08^{* * *}$ \\
\hline & High-SCH & $19.78 \pm 8.18$ & $26.10 \pm 10.90 * *$ & $26.98 \pm 11.24 * * *$ & $28.66 \pm 12.28 * * *$ \\
\hline \multirow[t]{4}{*}{ IVSd (mm) } & Control & $7.76 \pm 0.94$ & $6.78 \pm 0.90^{*}$ & $6.69 \pm 0.63 *$ & $5.91 \pm 0.96^{* * *}$ \\
\hline & Pimobendan & $6.30 \pm 2.76$ & $6.73 \pm 2.87$ & $6.78 \pm 2.98$ & $6.02 \pm 2.88$ \\
\hline & Low-SCH & $6.87 \pm 3.12$ & $5.30 \pm 2.22 *$ & $5.05 \pm 2.11^{* *}$ & $5.66 \pm 2.41$ \\
\hline & High-SCH & $7.32 \pm 3.35$ & $6.70 \pm 2.95$ & $6.70 \pm 2.97$ & $5.66 \pm 2.34$ \\
\hline \multirow[t]{4}{*}{ IVSs (mm) } & Control & $11.16 \pm 1.52$ & $9.04 \pm 1.02 *$ & $8.83 \pm 0.52 * *$ & $8.43 \pm 1.17 * *$ \\
\hline & Pimobendan & $9.89 \pm 4.43$ & $9.14 \pm 3.91$ & $9.15 \pm 4.25$ & $8.74 \pm 3.73$ \\
\hline & Low-SCH & $9.75 \pm 4.25$ & $7.85 \pm 3.30^{*}$ & $7.37 \pm 3.10^{* *}$ & $7.40 \pm 3.10^{* *}$ \\
\hline & High-SCH & $11.47 \pm 5.01$ & $9.72 \pm 4.12$ & $9.44 \pm 4.06^{*}$ & $8.99 \pm 3.73 *$ \\
\hline \multirow[t]{4}{*}{ LVPWd (mm) } & Control & $7.82 \pm 1.35$ & $5.96 \pm 1.13^{*}$ & $6.20 \pm 1.44$ & $6.67 \pm 2.43$ \\
\hline & Pimobendan & $6.31 \pm 2.67$ & $6.61 \pm 2.79$ & $6.43 \pm 2.98$ & $6.34 \pm 2.91$ \\
\hline & Low-SCH & $7.15 \pm 3.16$ & $6.35 \pm 2.67^{*}$ & $6.33 \pm 2.68^{*}$ & $5.96 \pm 2.49^{* *}$ \\
\hline & High-SCH & $7.56 \pm 3.25$ & $5.79 \pm 2.58^{*}$ & $5.06 \pm 2.13 * *$ & $5.13 \pm 2.19^{* *}$ \\
\hline \multirow[t]{4}{*}{ LVPWs (mm) } & Control & $10.60 \pm 1.64$ & $7.16 \pm 1.52 * * *$ & $6.78 \pm 1.72 * * *$ & $7.28 \pm 2.54 * * *$ \\
\hline & Pimobendan & $10.46 \pm 4.60$ & $8.42 \pm 3.65$ & $8.85 \pm 4.18$ & $8.60 \pm 3.68$ \\
\hline & Low-SCH & $10.58 \pm 4.64$ & $8.40 \pm 3.66^{* *}$ & $7.89 \pm 3.30 * *$ & $7.96 \pm 3.30 * *$ \\
\hline & High-SCH & $10.07 \pm 4.15$ & $7.61 \pm 3.26^{* * *}$ & $7.21 \pm 3.10^{* * *}$ & $6.79 \pm 3.32 * * *$ \\
\hline \multirow[t]{4}{*}{ FS (\%) } & Control & $34.70 \pm 4.60$ & $19.90 \pm 4.00 * * *$ & $18.60 \pm 1.40^{* * *}$ & $17.80 \pm 0.80^{* * *}$ \\
\hline & Pimobendan & $37.25 \pm 16.84$ & $23.03 \pm 10.20^{*}$ & $24.46 \pm 14.87^{*}$ & $23.68 \pm 11.09$ \\
\hline & Low-SCH & $36.58 \pm 15.69$ & $27.16 \pm 11.72 * *$ & $23.72 \pm 10.27 * * *$ & $22.72 \pm 9.56^{* * *}$ \\
\hline & High-SCH & $35.48 \pm 14.60$ & $23.16 \pm 10.24 * * *$ & $24.86 \pm 10.74 * * *$ & $23.92 \pm 11.92 * * *$ \\
\hline
\end{tabular}

Variables are expressed as the mean \pm standard deviation. LVIDd, left ventricular end diastolic diameter; LVIDs, left ventricular end systolic diameter; IVSd, diastolic interventricular septal thickness; IVSs, systolic interventricular septal thickness; LVPWd, diastolic left ventricular free wall thickness; LVPWs, systolic left ventricular free wall thickness; FS, fractional shortening. $* P<0.05, * * P<0.01, * * * P<0.001 \mathrm{vs}$. corresponding baseline values.

\section{Two-dimensional tissue tracking}

In the control, pacing had resulted in significant reduction of global strains in both radial and circumferential directions from week 1 (Table 2). Regionally, reduction of strains were observed in all six myocardial segments in both directions by the week 3 of pacing $(P<0.02$ for radial and $P<0.01$ for circumferential directions), with the exception of the anterior septal segment, in which no significant changes were observed. Moreover, significant increase in STI was seen from week 1.

Similarly in Low- and High-SCH groups, global strains in both directions resulted in significant reduction from week 1 of pacing, except for circumferential strain in the Low-SCH group, which was only observed from week 2 . Regionally in the Low-SCH group, significant reductions of strains were observed in radial anterior septal
$(P=0.02)$, posterior $(P=0.01)$, and inferior segments $(P=0.01)$ by week 3 of pacing, and no changes were observed in the circumferential directions. In the High$\mathrm{SCH}$ group, significant reductions of strains were observed in radial posterior $(P=0.05)$, inferior $(P=0.01)$, and septal segments $(P=0.02)$ and in circumferential anterior $(P=0.02)$ and septal segments $(P=0.004)$ by week 3. For STI, while no significant change was observed in the Low-SCH group, significant increase was observed in the High-SCH group from week 2.

On the other hand, in the pimobendan group, significant reduction of global strain was observed in the radial direction only. Regionally, anterior septal segments in radial $(P=0.009)$ and circumferential direction $(P=0.04)$ showed significant reduction from week 2 and only at week 2 , respectively, and no other segments showed any significant changes. Similar to the control 
Table 2. Temporal comparison of the global strain and synchronicity parameter in control, and with the administration of pimobendan $(0.25 \mathrm{mg} / \mathrm{kg}$ PO q12hrs) and two doses of SCH00013 (1 and $3 \mathrm{mg} / \mathrm{kg}$ PO q12hrs) on of canine pacing-induced heart failure model

\begin{tabular}{llcccc}
\hline & & Baseline & Week 1 & Week 2 & Week 3 \\
\hline Radial strain (\%) & Control & $31.96 \pm 7.12$ & $22.62 \pm 4.98^{* *}$ & $17.88 \pm 6.62^{* * *}$ & $13.52 \pm 3.49^{* * *}$ \\
& Pimobendan & $32.72 \pm 17.63$ & $19.56 \pm 10.95^{*}$ & $20.63 \pm 9.15^{*}$ & $20.78 \pm 12.46^{*}$ \\
& Low-SCH & $42.46 \pm 19.29$ & $27.72 \pm 12.32^{* *}$ & $21.95 \pm 9.85^{* * *}$ & $25.44 \pm 11.99^{* *}$ \\
& High-SCH & $50.47 \pm 22.79$ & $27.40 \pm 14.89^{*}$ & $27.20 \pm 15.38^{*}$ & $26.38 \pm 17.11^{*}$ \\
Circumferential & Control & $-15.44 \pm 1.50$ & $-11.60 \pm 2.95^{* *}$ & $-9.32 \pm 1.61^{* * *}$ & $-7.62 \pm 1.47 * * *$ \\
strain (\%) & Pimobendan & $-15.53 \pm 6.48$ & $-11.99 \pm 5.89$ & $-11.31 \pm 5.42$ & $-12.24 \pm 5.32$ \\
& Low-SCH & $-15.71 \pm 6.80$ & $-12.61 \pm 5.39$ & $-12.25 \pm 5.42^{*}$ & $-11.50 \pm 5.13^{*}$ \\
STI (ms) & High-SCH & $-19.43 \pm 7.95$ & $-8.55 \pm 4.91 * *$ & $-11.03 \pm 5.13^{*}$ & $-11.36 \pm 6.18^{*}$ \\
& Control & $44.25 \pm 17.60$ & $60.50 \pm 10.93$ & $84.57 \pm 34.04^{*}$ & $107.40 \pm 37.31^{* * *}$ \\
& Pimobendan & $36.42 \pm 10.83$ & $61.29 \pm 16.36^{*}$ & $64.29 \pm 15.84^{*}$ & $71.44 \pm 18.39^{* *}$ \\
& Low-SCH & $55.30 \pm 8.27$ & $56.61 \pm 19.06$ & $62.46 \pm 13.09$ & $65.07 \pm 7.64$ \\
& High-SCH & $49.44 \pm 5.34$ & $58.26 \pm 12.51$ & $78.73 \pm 25.85^{*}$ & $81.33 \pm 19.66^{*}$ \\
\hline
\end{tabular}

Variables are expressed as the mean \pm standard deviation. STI, synchronisity time index. $* P<0.05, * * P<0.01, * * * P<0.001$ vs. corresponding baseline values.

and High-SCH groups, the pimobendan group showed significant increase of STI, which was observed from week 1 of pacing.

Between groups, significant difference was observed in global circumferential strain between the control and pimobendan group at week $3(P=0.02)$. Regionally, anterior and lateral segments showed significant differences in circumferential direction. The anterior segment $(P=0.007)$ showed a significant difference between the control and Low-SCH group from week 2 and between the pimobendan group and control and High$\mathrm{SCH}$ group at week 3. For the lateral segment $(P=0.02)$, a significant difference was seen between the control and pimobendan group at week 3 .

\section{Discussion}

In this study, the effect of oral administration of pimobendan and SCH00013 on the myocardial function of the canine pacing-induced HF model was evaluated using echocardiography. From past reports, sustained high-electrical pacing has been shown to produce dilated, congestive cardiomyopathy in dogs, and this model of HF has been widely used to study CHF $(15-18)$. In the control of this study, 3 weeks of sustained highelectrical pacing at the rate of $250 \mathrm{bpm}$ had resulted in $\mathrm{HF}$, characterized by thinning and dilation of LV, and impaired systolic function indicated by the reduction of FS and strain values. Additionally, diastolic dysfunction consistent with impaired compliance and LV dyssynchrony were also observed.

Administrations of pimobendan $(0.25 \mathrm{mg} / \mathrm{kg}$, i.v. $)$ and $\mathrm{SCH} 00013(0.3-3 \mathrm{mg} / \mathrm{kg}$, i.v. $)$ in the canine pacinginduced HF model are known to elicit PIE by increasing the maximum rate of rise of $\mathrm{LV}$ pressure $\left(\mathrm{LVdP} / \mathrm{dt}_{\max }\right)$ (7, 19). In this study, significant echocardiographic changes indicative of LV morphological change were observed in all treatment groups, but pimobendan showed the least LV structural changes. Additionally, even though impairment of the systolic function was observed in all treatment groups, global strain analysis revealed that administration of pimobendan was able to sustain myocardial function in the circumferential direction, where SCH00013 could not. Such a result may suggest pimobendan to have a stronger PIE in comparison to SCH00013. Interestingly, regional strain analysis revealed less segments to be impaired on the circumferential direction in comparison to the radial direction in all treatment groups. Circumferential LV mechanics is governed by the midmyocardial and epicardial function, whereas radial LV mechanics is mostly governed by the midmyocardial function (20). Therefore, these results may suggest the effect of the treatment to be the greatest at the epicardium.

Intravenous and oral administration of $\mathrm{SCH} 00013$ $(0.3-10 \mathrm{mg} / \mathrm{kg})$ in normal dogs are also known to elicit PIE in a dose-dependent manner, shown by the increase of $\mathrm{LVdP} / \mathrm{dt}_{\max }$ (11). Similarly, dose-dependent increase in $\mathrm{LVdP} / \mathrm{dt}_{\max }$ is observed with intravenous administration of SCH00013 $(0.3-3 \mathrm{mg} / \mathrm{kg})$ in the canine pacinginduced HF model (19). The results of this study showed progressive deterioration of systolic function, demonstrated by significant reduction of FS and global strains in both SCH00013-treated groups. Regional strain analysis revealed myocardial impairments in both directions in the High-SCH group, whereas the Low$\mathrm{SCH}$ group showed regional myocardial impairment only in the radial direction. Such results indicate the 
low dose of SCH00013 was better able to sustain PIE, a result contrary to the previous reports.

PIE of SCH00013 is produced mainly by the increased calcium sensitivity, and partially by the PDE III inhibition, but PDE III inhibition of SCH00013 increases with increased concentration $(10,12)$. This is supported by the evidence of decreased BP observed with high dose of SCH00013, which was also seen in this study (11). Additionally, failing myocardium in CHF is known to have impaired response to PDE III inhibitors, due to reduced basal cAMP formation (5). Therefore, increased PDE III inhibitory action, together with impaired response to PDE III inhibitors in HF, might have resulted in failure to sustain PIE at high dose of SCH00013 in this study. The results of this study have also shown that pimobendan was better able to sustain PIE in HF. Similar results have been seen where pimobendan enabled PIE to persist in CHF, which is thought to be due to the effect of calcium sensitization (7). Comparable results have been seen with levosimendan, another calcium sensitizer with similar property to pimobendan (21).

LV mechanical dyssynchrony is known to be a sensitive indicator of myocardial dysfunction, and increased dyssynchrony has been shown to correlate with severity of LV function in patients with dilated cardiomyopathy $(22-24)$. For such reasons, it has been widely used for patient selection and assessment of the response to cardiac resynchronization therapy $(20,22,25)$. In this study, significant LV dyssynchrony was observed in both pimobendan and High-SCH groups. On the contrary, the Low-SCH group was able to maintain LV synchronicity. This may suggest that even though the PIE of SCH00013 may not be as potent as pimobendan, low dose of SCH00013 also has a beneficial effect on the LV myocardial function. Additionally, such results allow us to further speculate that this ability to sustain LV synchronicity at low dose SCH00013 may be due to the effect of the calcium sensitization.

Calcium sensitizers with concomitant PDE III inhibitory action possess a hypotensive effect due to its vasodilatory action of PDE III inhibition and reduction of BP with the administration of pimobendan has been reported $(5,26)$. However, in this study, administration of pimobendan did not result in reduced BP. Similar results have been observed by Ohte et al., where administration of pimobendan $(0.25 \mathrm{mg} / \mathrm{kg}$, i.v. $)$ to dogs with pacing-induced HF did not reduce the end systolic BP because of the markedly increased stroke volume $(7,27)$. Species differences in vasodilatory effect of pimobendan has been reported, where pimobendan had a more potent cardiac effect than vascular effect (26).

Limitations of this study include the small number of animals per group. Lack of statistical difference between treatment groups could perhaps be due to the study population. Additionally, parameters such as FS and strain values are influenced by the loading condition. Therefore, when evaluating the result of these parameters, factors influencing the loading conditions, such as changes in blood pressure, should be taken into considerations.

Results of this study demonstrated the possibility of varying effect of pimobendan and SCH00013 on the myocardial function in the canine pacing-induced $\mathrm{HF}$ model. Additionally, the effect of SCH00013 on the myocardium differed depending on the dose, most likely caused by the different proportion of calcium sensitizing and PDE II-inhibiting actions. SCH00013 has a higher proportion of calcium-sensitizing action, and in this study, low dose of SCH00013 showed sustained LV synchronicity, although PIE was not as potent. However, at a higher dose, the action of PDE III inhibition predominated, which was suggested by the reduction of $\mathrm{BP}$, and this resulted in deterioration of both PIE and LV synchronicity. The results have suggested pimobendan to have a potent PIE on the pacing-induced progressive HF, but little effect on the LV dyssynchrony. On the other hand, although the PIE is not as potent, low dose of SCH00013 is thought to be effective in sustaining LV synchronicity. Although, significant statistical differences between pimobendan and $\mathrm{SCH} 00013$ were not observed, the results of this study allow speculations that administration of pimobendan and $\mathrm{SCH} 00013$ may have differing effect on the myocardial function in the canine pacing-induced HF model. This lack of statistical differences could be due to the small number of animals per group; therefore, a larger study should be considered.

\section{Acknowledgments}

Pimobendan and SCH00013 were generously donated by Nippon Boehringer Ingelheim Co., Ltd. and Zenyaku Kogyo Co., Ltd. respectively.

\section{Conflicts of Interest}

We state that none of the authors of this manuscript have any conflicts of interest.

\section{References}

1 Metra M, Bettari L, Carubelli V, Cas LD. Old and new intravenous inotropic agents in the treatment of advanced heart failure. Prog Cardiovasc Dis. 2011;54:97-106.

2 Perrone SV, Kaplinsky EJ. Calcium sensitizer agents: a new class of inotropic agents in the treatment of decompensated heart failure. International journal of cardiology. 2005;103:248-255.

3 Endoh M. Cardiac Ca2+ signaling and $\mathrm{Ca} 2+$ sensitizers. Circ J. 
2008;72:1915-1925.

4 Nielsen-Kudsk JE,Aldershvile J. Will calcium sensitizers play a role in the treatment of heart failure? J Cardiovasc Pharmacol. 1995;26 Suppl 1:S77-S84.

5 Bohm M, Morano I, Pieske B, Ruegg JC, Wankerl M, Zimmermann R et al. Contribution of cAMP-phosphodiesterase inhibition and sensitization of the contractile proteins for calcium to the inotropic effect of pimobendan in the failing human myocardium. Circ Res. 1991;68:689-701.

6 Matsui K, Kiyosue T, Wang JC, Dohi K, Arita M. Effects of pimobendan on the L-type $\mathrm{Ca} 2+$ current and developed tension in guinea-pig ventricular myocytes and papillary muscle: comparison with IBMX, milrinone, and cilostazol. Cardiovasc Drugs Ther. 1999;13:105-113.

7 Ohte N, Cheng CP, Suzuki M, Little WC. The cardiac effects of pimobendan (but not amrinone) are preserved at rest and during exercise in conscious dogs with pacing-induced heart failure. J Pharmacol Exp Ther. 1997;282:23-31.

8 Fujino K, Sperelakis N, Solaro RJ. Sensitization of dog and guinea pig heart myofilaments to $\mathrm{Ca} 2+$ activation and the inotropic effect of pimobendan: comparison with milrinone. Circ Res. 1988;63:911-922.

9 Endoh M, Sugawara H, Mineshima M. Pharmacology of SCH00013: a novel Ca2+ sensitizer. Cardiovasc Drug Rev. 2001;19:345-366.

10 Hino M, Sugawara H, Yoshimura A, Ogura A, Yoshioka K, Sakato M, et al. Investigation on SCH00013, a novel cardiotonic agent with $\mathrm{Ca}++$ sensitizing action. 1st communication: phosphodiesterase III inhibitory effect and class III antiarrhythmic effect in guinea-pig heart. Arzneimittelforschung. 1999;49:398-406.

11 Yoshimura A, Koide Y, Yoshioka K, Hino M, Sugawara H, Sakato M, et al. Investigation on SCH00013, a novel cardiotonic agent with $\mathrm{Ca}++$ sensitizing action. 2 nd communication: in vivo cardiovascular effects and bioavailability in dogs. Arzneimittelforschung. 1999;49:407-411.

12 Sugawara H, Endoh M. A novel cardiotonic agent SCH00013 acts as a $\mathrm{Ca}++$ sensitizer with no chronotropic activity in mammalian cardiac muscle. J Pharmacol Exp Ther. 1998;287: 214-222.

13 Hamabe L, Fukushima R, Kawamura K, Shinoda Y, Che HH, Suzuki S, et al. Evaluation of changes in left ventricular myocardial function observed in canine myocardial dysfunction models using two-dimensional tissue tracking technique. J Vet Sci. 2013;14:355-362.

14 Boon J. The M-mode and Doppler examination. Veterinary Echocardiography. 2nd ed. Chichester: Wiley-Blackwell, 2011: 101-112.

15 Wilson JR, Douglas P, Hickey WF, Lanoce V, Ferraro N, Muhammad A, et al. Experimental congestive heart failure produced by rapid ventricular pacing in the dog: cardiac effects.
Circulation. 1987;75:857-867.

$16 \mathrm{Wu}$ W, Wang H, Tang Y, Yuan W, Jiang Y. Application of quantitative tissue velocity imaging to evaluate left ventricular early diastolic dysfunction in dogs with heart failure due to rapid ventricular pacing. J Am Soc Echocardiogr. 2008;21:1269-1276.

17 Zupan I, Rakovec P, Budihna N, Brecelj A, Kozelj M. Tachycardia induced cardiomyopathy in dogs; relation between chronic supraventricular and chronic ventricular tachycardia. Int J Cardiol. 1996;56:75-81.

18 Shinbane JS, Wood MA, Jensen DN, Ellenbogen KA, Fitzpatrick AP, Scheinman MM. Tachycardia-induced cardiomyopathy: a review of animal models and clinical studies. J Am Coll Cardiol. 1997;29:709-715.

19 Tadano N, Morimoto S, Yoshimura A, Miura M, Yoshioka K, Sakato M, et al. SCH00013, a novel $\mathrm{Ca}(2+)$ sensitizer with positive inotropic and no chronotropic action in heart failure. J Pharmacol Sci. 2005;97:53-60.

20 Geyer H, Caracciolo G, Abe H, Wilansky S, Carerj S, Gentile F, et al. Assessment of myocardial mechanics using speckle tracking echocardiography: fundamentals and clinical applications. J Am Soc Echocardiogr. 2010;23:351-69; quiz 453-455.

21 Masutani S, Cheng HJ, Hyttila-Hopponen M, Levijoki J, Heikkila A, Vuorela A, et al. Orally available levosimendan dose-related positive inotropic and lusitropic effect in conscious chronically instrumented normal and heart failure dogs. J Pharmacol Exp Ther. 2008;325:236-247.

22 Dandel M, Hetzer R. Echocardiographic strain and strain rate imaging--clinical applications. International journal of cardiology. 2009;132:11-24.

23 Labombarda F, Blanc J, Pellissier A, Stos B, Gaillard C, Bajolle F, et al. Health-e-Child project: mechanical dyssynchrony in children with dilated cardiomyopathy. J Am Soc Echocardiogr. 2009;22:1289-1295.

24 Friedberg MK, Slorach C. Relation between left ventricular regional radial function and radial wall motion abnormalities using two-dimensional speckle tracking in children with idiopathic dilated cardiomyopathy. Am J Cardiol. 2008;102: 335-339.

25 Nesbitt GC, Mankad S, Oh JK. Strain imaging in echocardiography: methods and clinical applications. Int $\mathrm{J}$ Cardiovasc Imaging. 2009;25 Suppl 1:9-22.

26 Duncker DJ, Hartog JM, Levinsky L, Verdouw PD. Systemic haemodynamic actions of pimobendan (UD-CG 115 BS) and its O-demethylmetabolite UD-CG $212 \mathrm{Cl}$ in the conscious pig. $\mathrm{Br} \mathrm{J}$ Pharmacol. 1987;91:609-615.

27 Suzuki S, Fukushima R, Ishikawa T, Hamabe L, Aytemiz D, Huai-Che $\mathrm{H}$, et al. The effect of pimobendan on left atrial pressure in dogs with mitral valve regurgitation. J Vet Intern Med. 2011;25:1328-1333. 